\title{
A NOVEL APPROACH TO FAULT DETECTION AND DIAGNOSIS ON WIND TURBINES
}

\author{
GÓMEZ MUÑOZ C.Q. ${ }^{1}$ \\ RUIZ DE LA HERMOSA GONZÁLEZ-CARRATO R. ${ }^{2, *}$ \\ TRAPERO ARENAS J.R ${ }^{3}$ \\ GARCÍA MÁRQUEZ F.P. ${ }^{1}$
}

\author{
${ }^{1}$ Ingenium Research Group, ETSII, \\ Avda. Camilo José Cela S/N. 13071 Ciudad Real, Spain \\ ${ }^{2}$ Colegio Universitario de Estudios Financieros \\ Serrano Anguita, 8. 28009 Madrid, Spain \\ ${ }^{3}$ Facultad de Ciencias y Tecnologías Químicas \\ Avda. Camilo José Cela S/N, 13071 Ciudad Real, Spain
}

Received: 30/01/2014

Accepted: 18/03/2014

Available online: 13/05/2014

*to whom all correspondence should be addressed: e-mail: raulruiz@cunef.edu

\section{ABSTRACT}

The structure of the wind turbines nowadays is a critical element due to their importance from the reliability, availability, safety, and cost points of view. This is more relevant when the offshore wind turbine is considered. This paper introduces a novel design of a Fault Detection and Diagnosis (FDD) model based on ultrasound technique. The FDD model will be able to detect fault/failures via the pulseecho technique. The pulse-echo is got via piezoelectric transducers that are also employed as sensors. The signal processing is based on two steps. Firstly, a wavelet transform is applied to the measured signals with filtering purposes, in order to enhance the signal to noise ratio. Secondly, a time series modeling approach, as an autoregressive with exogenous input model, is employed for pattern recognition by minimizing the Akaike information criterion. An experimental platform is proposed to test the procedure, where pulse-echo experiments were employed before and after a fault occurred. The results from this paper lead to the identification of an early indication of structural problems induced by internal (material, shape, age, etc.) and external (temperature, humidity, pressure, etc.) factors. The model can anticipate catastrophic faults, reducing the preventive/corrective tasks and costs, etc, and increasing the availability of the wind turbine, and therefore the energy production.

Keywords: Signal processing, transfer function, condition monitoring, wavelet transform, ARX models, fault detection and diagnosis, ultrasounds.

\section{Introduction}

Non-destructive testing (NDT) has become an essential technique for the development of structure health monitoring (SHM) systems. NDT use is increasing in many scientific and industrial fields, from wind energy production (García et al., 2012) to the transportation of gases and liquids (Llamas et al., 2014). The NDT progress depends on the continuous changes of the industry to suit the current scenarios. The benefits that these new techniques provide are related to the improvement of the product quality, public safety and especially the prevention of faults. The main consequence is the reduction of costs, since they can anticipate faults that involve both material and human losses to reduce the corrective/preventive maintenance tasks, and to increase the life cycle of the structure. Other advantages that they present are related to the forecasting analysis based on the data acquired in a real-time mode, as well as the establishment of FDD techniques. 
Within the NDT field, guided ultrasonic waves are one of the most prominent areas of exploitation. These waves are especially useful in geometries such as plates or tubes, since their finite geometry enables energy flow through transversal sections. These waves can travel long distances, allowing inspections of large areas without any relocation of the actuator, as in classical ultrasonic methods (Dai et al., 2014).

Ultrasonic waves are particularly appropriate for the monitoring of inaccessible areas, e.g. partially buried structures, isolated materials, corrosive atmospheres or structures under the sea. The absence of risks for the operator, equipment portability, and the achievement of fast results are also factors to be considered (Wang et al., 2010).

The purpose of this paper is to design a FDD model using ultrasound inputs in conjunction with advanced signal processing methods to monitor the structural assessment of wind turbines. This system will be able to detect faults or structural modifications e.g. scratches, cuts, changes in thickness or edges by identifying pattern changes in the pulse-echo signals. Note that pattern changes are associated to the aforementioned potential faults. The signal processing is based on the wavelet transform as a filtering technique and an autoregressive exogenous (ARX) model to estimate potential faults, where the exogenous variable is the input signal. An experimental platform is designed in order to illustrate the results. A steel plate is used as a test bed. The plate features may simulate the behavior of certain parts of wind turbines.

The article is organized as follows: Section 2 describes the experimental platform. Section 3 introduces the wavelet and ARX models. Experimental results are detailed in Section 4. Section 5 proposes a framework to diagnose the different kind of faults; and finally, the main conclusions are drawn in Section 6.

\section{Laboratory platform}

Different experiments were carried out in an experimental platform to obtain the proposed FDD. The platform (see Figure 1) consists of a device that is able to read and generate signals at $4 \mathrm{MS} \mathrm{s}^{-1}$. The device is connected to a PC for condition monitoring. The output signal from the device goes through an amplifier to drive the piezoelectric transducers. The high frequency amplifier is used to enhance the signal to noise ratio. In this particular case, the Hanning window is employed for the pulse inputs. The main advantage of the Hanning window is its low aliasing and loss of resolution. Furthermore, it reduces the generation of side lobes in its frequency spectrum.

The piezoelectric transducers used are macro-fiber composites (MFC) working as actuators and sensors.

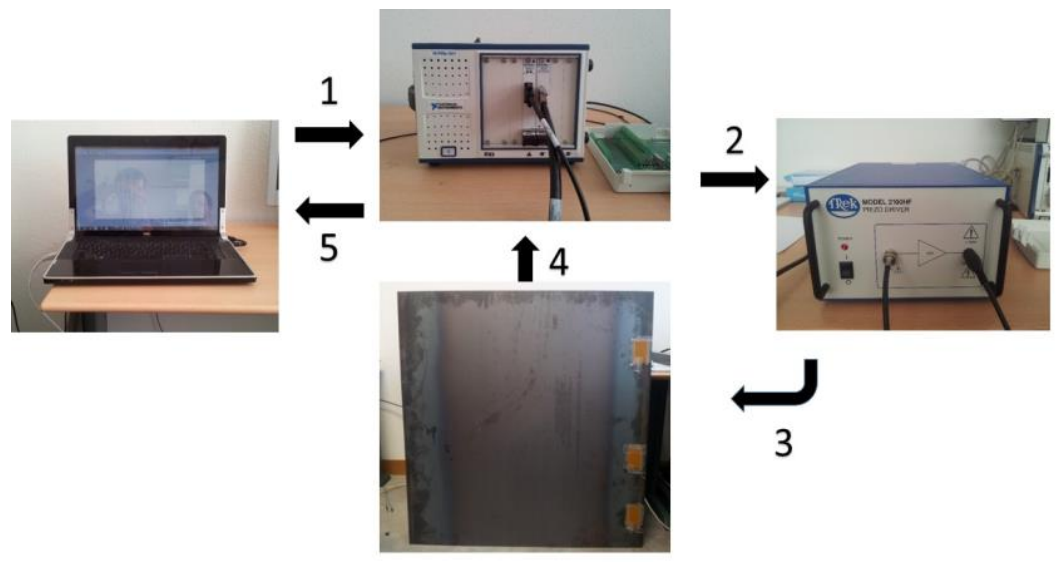

Figure 1. Laboratory NDT

The experiment procedure can be summarized as follows: a Hanning pulse is generated from the computer and sent to the MFC. It emits a pulse in the form of vibrations that flow through the structure to be inspected and is read by the other MFCs. This technique is called the transmission method (Chen, 
2010). If there is a defect, for example a crack in the transmission direction of the wave, a reflection of part of the wave is received, and therefore, the delay in the wave transmission will be reduced and the piezoelectric sensor will detect that imperfection.

\section{Models}

The methodology to identify defects can be divided in two steps. Firstly, in order to enhance the signal to noise ratio and to avoid noise at different frequencies, a pre-filtering step based on a wavelet transform is proposed. Secondly, an ARX model is estimated to predict the output response for the baseline condition. This predicted output response can then be compared to the actual response measured during other experiments to determine whether a significant variation between both signals is present. In case that difference is important, the system is assumed to have changed in such a way that faults may be present (Light-Marquez et al., 2010). The rest of the section is devoted to introducing the wavelet and ARX methods, respectively.

\subsection{Wavelet transform}

Wavelet transform is a powerful tool applied to time and frequency domain analysis with the use of several decomposition coefficients at different frequency bands (Eristi, 2013). It leads to work with high and low frequencies, identifying spectral features, unusual temporary files, and lack of stationary and removing random noise (Dong et al., 2010). The wavelet transform improves the limitations of resolution; and the loss of information presented by the Short-Time Fourier Transform or the Fast Fourier Transform (Jia et al., 2003; Morsi et al, 2010).

Wavelet transforms are commonly categorized as continuous wavelet transforms (CWT), discrete wavelet transforms (DWT) or wavelet packet transforms (PWT) among others. Regardless of the categorization, all these types emerge from what is known as mother wavelet, which is given by the equation (1).

$\psi_{\mathrm{s}, \mathrm{\tau}}(\mathrm{t})=\left(\frac{1}{\sqrt{\mathrm{s}}}\right) \Psi\left(\frac{\mathrm{t}-\mathrm{\tau}}{\mathrm{s}}\right)$

where $s$ is the scale factor, and $\tau$ is the translational factor. The wavelet transform $W_{f}(s, \tau)$ of a function $f(t)$ is the decomposition of $f(t)$ in a set of functions forming a base with the conjugate of the mother wavelet $\left(\psi_{s, \mathrm{~T}}^{*}(\mathrm{t})\right)$. It is defined in equation $(2)$ :

$W_{f}(s, \tau)=\int f(t) \psi_{s, \tau}^{*}(t) d t$

The most recurrent families of wavelet transforms are Haar, Daubechies or Symlet transforms. The selection of a particular family can be set by the application where the wavelet is introduced. Daubechies wavelets are the most used wavelets, representing the foundations of wavelets signal processing. Daubechies wavelets lead more accurate results in comparison to other families (Patil et al., 2011; Genovese et al., 2008).

Research into leak detection in plastic pipes from acoustic emission signals (Ahadi et al., 2010), cracks (Ye et al., 2010) or evaluation of the corrosion on non-accessible pipes can be found (Acciani et al., 2010) based on wavelet transforms.

\section{$3.2 \operatorname{Arx}$}

Time series methods have been previously employed for failure prediction and detection. For instance, (Garcia et al., 2010) and (Pedregal et al., 2010) utilize a VARMA (vector auto-regressive moving-average) and harmonic regressions, respectively, for failure prediction in railway elements. Here, an ARX model is proposed to identify faults in wind turbines elements. Basically, an ARX model can be expressed by a linear difference equation, such as (3):

$y_{t}+a_{1} y_{1}+\ldots+a_{n a} y_{t-n a}=b_{1} u_{t-n k}+\ldots+b_{n b} u_{t-n k-n b+1}+\varepsilon_{t}$ 
where AR refers to the autoregressive part and $X$ to the extra input, sometimes called the exogenous variable. The parameters $n_{a}$ and $n_{b}$ are the orders of the ARX model, and $n_{k}$ is the number of input samples that occur before the input affects the output, also called the delay in the system (Ljung, 2010). The variables $y_{t}$ and $u_{t}$ stand for the output and input responses, respectively. Model orders $n_{a}, n_{b}$ and $n_{k}$ have been chosen by minimizing the Akaike's Information Criterion (AIC) (Akaike, 1974). Model selection and the estimation of the unknown parameters $a_{i}, i=1, \ldots, n_{a}$ and $b_{j}, j=1, \ldots, n_{b}$ have been done by means of the routines implemented in the MATLAB ${ }^{\mathrm{TM}}$ System Identification toolbox (Ljung, 2012).

\section{Experimental results}

To achieve the objectives of the case study, several experiments on a free fault plate are carried out. They are then repeated after making a cut over the entire thickness of the plate. Distances and measurement conditions remain unchanged in order to analyse the behaviour of the material in both circumstances.

The square steel plate has a length of $106 \mathrm{~cm}^{2}$ and a thickness of $1.2 \mathrm{~mm}$. Three MFC transducers are located as shown in Figure 2. The first sensor located at $14 \mathrm{~cm}$ from the left extreme acts as actuator; and the remaining as sensors. The emitted signal will be a Hanning pulse at $25 \mathrm{kHz}, 2$ cycles and a sampling time of 40,000 samples per second (see Figure 3). The input frequency is set to $25 \mathrm{kHz}$ to achieve a compromise between good response and MFC limitations when they work as actuators. Additionally, the pulse is composed of two cycles, in order to create a narrow pulse and to avoid undesired overlaps between the emitted pulse and the first received echoes.

The received signals are recorded and the pattern between both sensors is analysed. Next, the different cuts between the second and the third sensor are made as is depicted in the right panel of Figure 2 . Then, the same input signal is applied to determine how the pattern has changed with regards to the initial fault-free experiment.
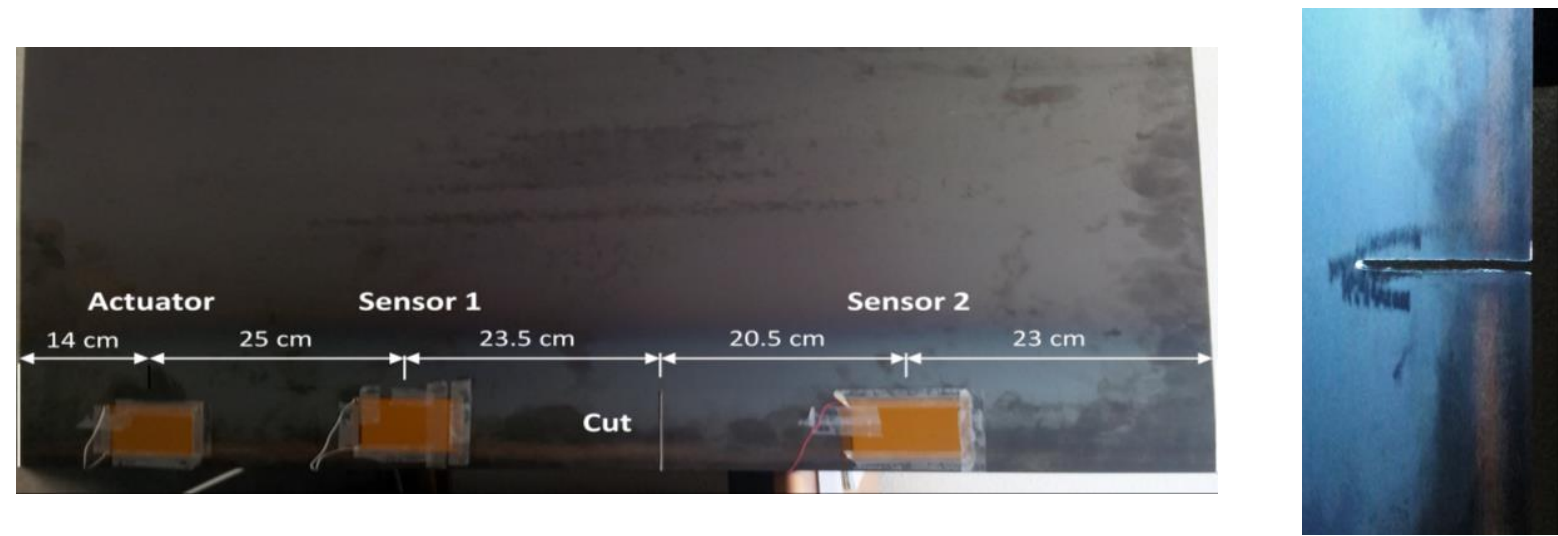

Figure 2. Actuator and sensors placement (left). Zoom of the cut (right)

It should be pointed out that the ARX model uses as input $u$ in (3) the observations measured in the sensor 1 ( $25 \mathrm{~cm}$ to the right of the actuator) and as output the sensor 2 closer to the right extreme.

A MATLAB ${ }^{T M}$ code is implemented to pre-filter the signals. The inputs are the two original signals received by the sensors and the outputs are the filtered signals. The process is carried out by the discrete wavelet transform (DWT). DWT introduces a scale discretization given by equation (4) to reduce the redundant information from the continuous changes of the scale and translational parameters:

$W_{f}(i, j)=\frac{1}{\sqrt{2^{i}}} \int f(t) \psi^{*}\left(\frac{t-j 2^{i}}{2^{i}}\right) d t$

where $s=2^{i}$ and $\tau=k 2^{i}$ are dyadic scales. 


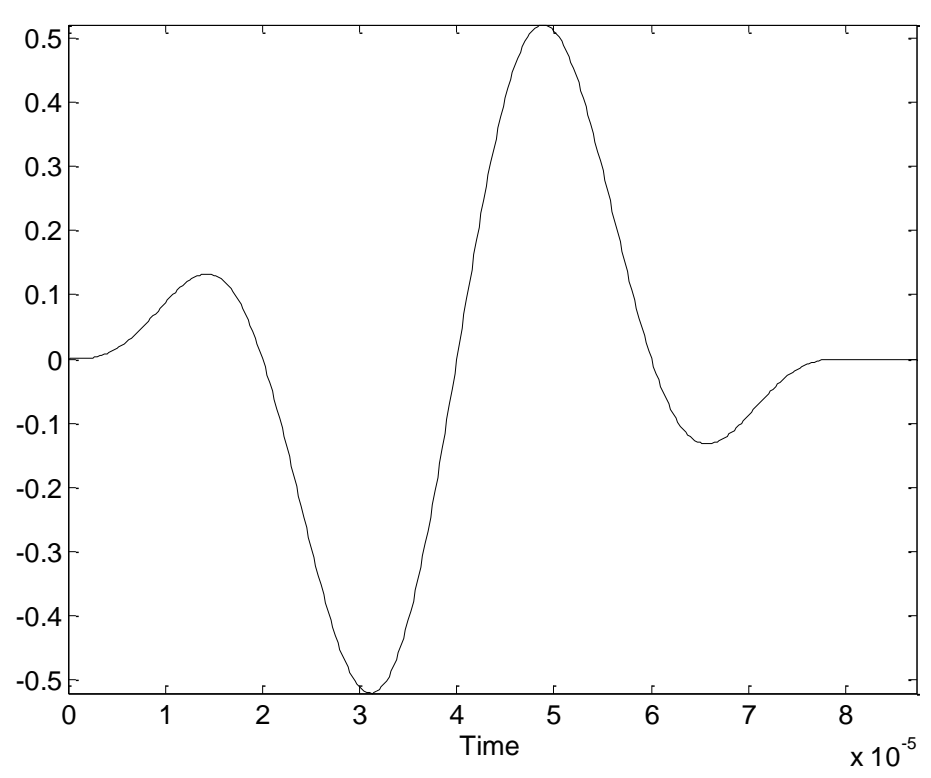

Figure 3. Hanning pulse at $25 \mathrm{KHz}$ with 2 cycles

The chosen family is the Daubechies wavelet transform. This is due to its versatility for processing signals presenting certain characteristics such as changes in shape and amplitude, obtaining more precise results than other wavelet families. An example of the signals without and with filtering is depicted in Figure 4. The technique also prevents border limitations, scaling errors or fidelity with a proper accuracy in the results. The approach is intended to avoid any type of noise complexity associated to the signal to be analysed.
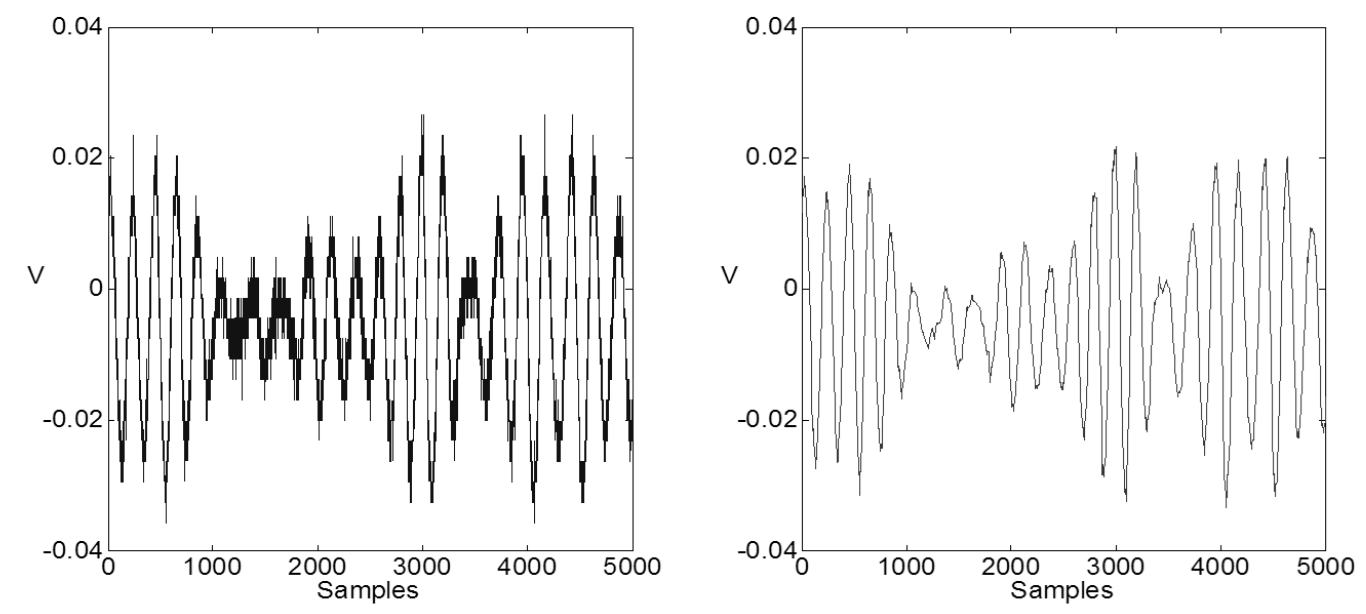

Figure 4. Unfiltered (left) vs. filtered signal (right)

To identify the ARX orders and to estimate their respective parameters, two experiments were carried out for the free-fault plate. The data of the first experiment was employed to identify and estimate an ARX model with $n_{a}=17 ; n_{b}=13$ and $n_{k}=20$, by minimizing the AIC criterion. The second experiment was used to validate the previous estimation.

Figure 5 shows the measured observations of that experiment validation, where the sensor 2 measurements are in solid line and the 20 step ARX model predicted output in dashed line. The Root Mean Squared Error (RMSE) associated to this predicted output is $2.27 * 10^{-4}$. 


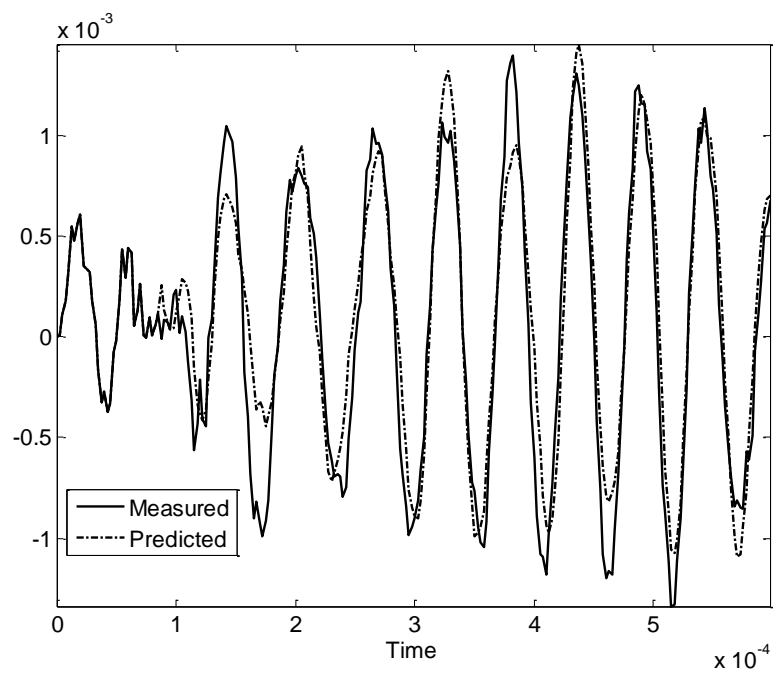

Figure 5. Measured (solid line) and 20 step predicted output (dashed line) for the free-fault case

As previously mentioned, the same input showed in Figure 3 was applied after a cut was made into the plate. Figure 6 shows again the measured and 20 step predicted output in solid and dashed line, respectively. However, in this case the model previously estimated is not adequate to reproduce the measured observations as a consequence of the fault. In fact, the RMSE is $4.45^{*} 10^{-4}$ which is significantly higher than the RMSE computed when no fault was present in the plate.

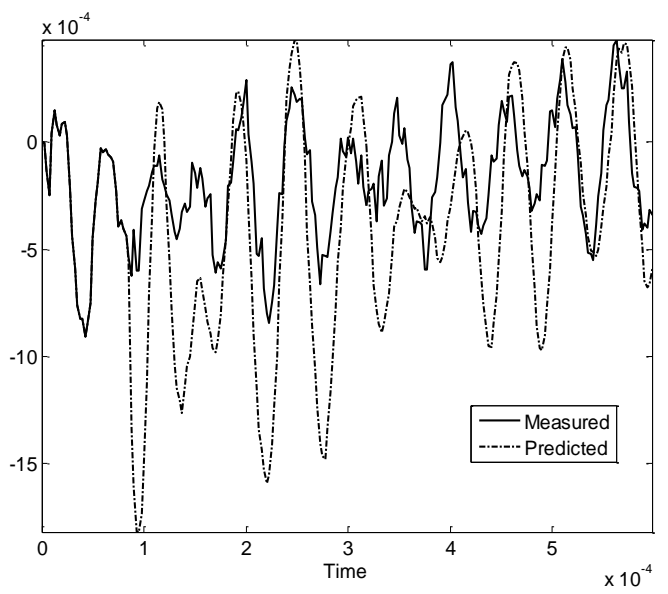

Figure 6. Measured (solid line) and 20 step predicted output (dashed line) when a fault has occurred

\section{Fault diagnosis}

The last section showed that the model proposed was able to detect a fault if a high RMSE was found in relation to the fault-free situation. Nonetheless, it would be more appealing from a practical point of view to be capable of detecting the potential fault before the complete system failure. In that sense, a third experiment has been designed, where the plate was partially cut $(8.5 \mathrm{~cm}$ long $\times 0.6 \mathrm{~mm}$ deep). Unlike the previous experiment, the plate was not cut over the entire thickness.

Figure 7 displays the RMSE found for each sensor. Upper and lower plot refer to the RMSE calculated for the sensor 1 and 2 (see sensor placement in Figure 2), respectively. Note that the RMSE was computed with respect to the free-fault experiment. Each bar in the figure corresponds to the average of the three experiments carried out under each condition. In other words, the fault-free experiment was repeated 
three times and the average RMSE was computed. Likewise, the early stage experiment (partial cut) was repeated three times. The average RMSE was calculated and the same procedure was applied to the crack case (entire thickness cut). The lower plot shows that the second and third bar provides a higher RMSE than the free-fault case. Therefore, the model is capable of detecting a fault or potential fault. Nevertheless, in order to distinguish whether the fault is at an early stage or it corresponds to a complete failure; the RMSE provided by the sensor 1 needs to be analyzed. Recall that the RMSE of the sensor 1 is the average of the RMSE computed with respect to the fault-free measured output of the same sensor 1 . Here, it should be pointed out that the sensor 1 RMSE is similar to the fault-free RMSE when a fault is at an early stage, whereas the RMSE is higher than the other two cases when a complete fault is present.

In summary, the results of sensor 2 inform about the presence of a fault in the structure, however, in order to distinguish whether that fault is at an early stage or a complete failure, information from sensor 1 should be analyzed. If RMSE in sensor 1 is higher than the fault-free RMSE a complete fault is found. Otherwise an early stage fault is emerging and maintenance activities should be carried out.

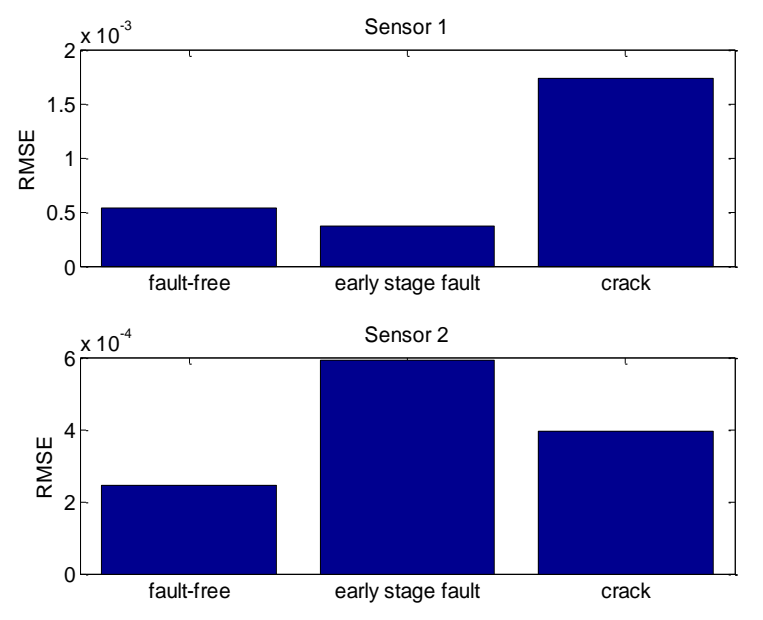

Figure 7. Root Mean Squared Error found for sensor 2 (upper plot) and sensor 1 (lower plot) between the measured sensor output and the free-fault baseline

In order to interpret these results, it is interesting to plot the measured output of sensor 2 (Figure 8) and sensor 1 (Figure 9) for each type of fault. When the plate starts to crack, Figure 8 shows how a delay in the sensor 2 early stage fault signal appears with respect to the free-fault case. However, when the plate suffers a complete crack, the sensor 2 does not receive the input signal and it mainly measures noise.

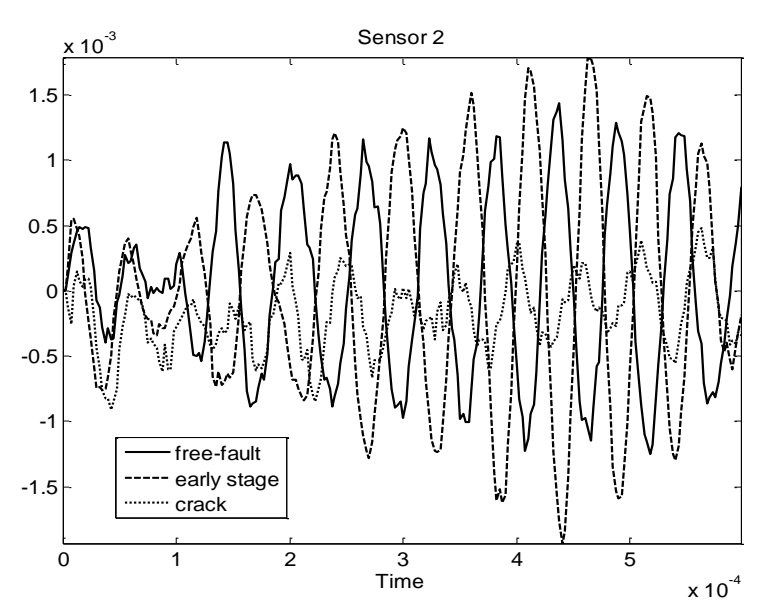

Figure 8. Sensor 2 measured signal for the free-fault (solid line), early stage (dashed line) and crack (dotted line) cases 
In addition to the information provided by sensor 2, it is important to analyze the sensor 1 output. Figure 9 shows when an early stage fault is present, how the signal goes through the material, and that the difference with respect to the fault-free test is negligible. On the other hand when the plate is completely cracked, the input signal does not go through the material and it is echoed back, yielding a totally different signal regarding the fault-free case.

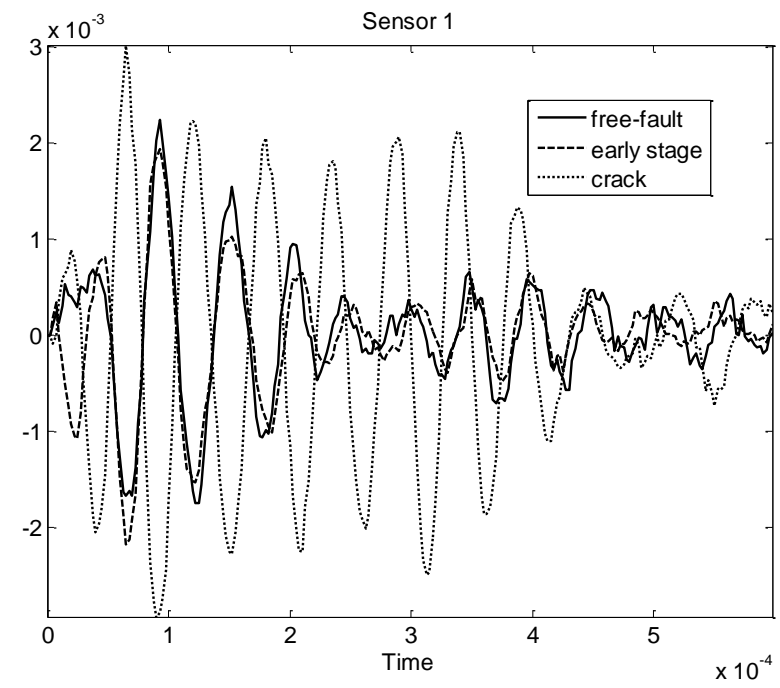

Figure 9. Sensor 1 measured signal for the free-fault (solid line), early stage (dashed line) and crack (dotted line) cases

\section{Conclusions}

NDT is considered one of the most effective condition monitoring methods nowadays. Its numerous benefits assure the safety and correct operation of devices, which results in reduction of costs and improvement of the lifecycle of these devices. In this sense, guided ultrasonic waves as a NDT technique are useful for their ability to monitor different geometries of limited access.

This paper reports the design of a FDD model using ultrasounds along with advanced signal processing tools: wavelet transform to filter the measured data and an ARX model to estimate potential faults. The system is capable of detecting structural changes by identifying pattern modifications in pulse-echo experiments. To achieve the intended objectives, several experiments were carried out on both a defect-free surface and on a damaged plate, with different extents of severity ranging from superficial cuts to a complete crack over the plate. The experimental results corroborate the adequacy of the proposed methodology to identify potential faults.

Further research should include the adequacy of the proposed procedure to identify other surface defects e.g. corrosion. Additionally, it should include other wind turbines parts such as wind turbine blades, taking into account material and superficial differences.

\section{Acknowledgments}

This work has been supported by the Spanish Ministerio de Economía y Competitividad, under Research Grant no. DPI2012-31579.

\section{References}

Acciani G., Brunetti G., Fornarelli G. and Giaquinto A. (2010), Angular and axial evaluation of superficial defects on non-accessible pipes by wavelet transform and neural network-based classification, Ultrasonics, 50(1), 13-25. 
Ahadi M. and Bakhtiar M.S. (2010), Leak detection in water-filled plastic pipes through the application of tuned wavelet transforms to acoustic emission signals, Applied Acoustics, 71(7), 634-639.

Akaike H. (1974), A new look at the statistical model identification, IEEE Transactions on Automatic Control, 19(6), 716-723.

Chen Y. (2010), Acoustical transmission line model for ultrasonic transducers for wide-bandwidth application, Acta Mechanica Solida Sinica, 23(2), 124-134.

Dai D. and He Q. (2014), Structure damage localization with ultrasonic guided waves based on a time-frequency method, Signal Processing, 96, 21-28.

Dong Y., Shi H., Luo J. and Fan G. and Zhang C. (2010), Application of wavelet transform in MCG-signal denoising, Modern Applied Science, 4(6), 20-24.

Eristi H. (2013), Fault diagnosis system for series compensated transmission line based on wavelet transform and adaptive neuro-fuzzy inference system, Measurement, 46 (1), 393-401.

Garcia F.P., Pedregal D.J. and Roberts C. (2010), Time series methods applied to failure prediction and detection, Reliability Engineering \& System Safety, 95(6), 698-703.

García F.P., Tobias A.M., Pinar J.M. and Papaelias M. (2012), Condition monitoring of wind turbines: Techniques and methods, Renewable Energy, 46, 169-178.

Genovese L., Neelov A., Goedecker S., Deutsch T., Ghasemi S., Willand A., Caliste D., Zilberberg O., Rayson M., Bergman A. and Schneider R. (2008), Daubechies wavelets as a basis set for density functional pseudopotential calculations, Journal of Chemical Physics, 129(1), 104-109.

Jia M. and Wang Y. (2003), Application of wavelet transformation in signal processing for vibrating platform, Journal of Shenyang Institute of Technology, 22(3), 53-55.

Light-Marquez A., Sobin A., Park G. and Farinholt K. (2011), Structural damage identification in wind turbine blades using piezoelectric active sensing, Structural Dynamics and Renewable Energy, 1, 55-65.

Ljung L. (2010), System identification, theory for the user. Second ed. Upper Saddle River, NJ: PTR Prentice Hall.

Ljung, L. (2012). System identification toolbox for use with Matlab (8th ed.). Natick, MA: The MathWorks, Inc., Version 8.

Llamas B., Mazadiego L.F., Elío J., Ortega M. F., Grandia F. and Rincones M. (2014), Systematic approach for the selection of monitoring technologies in $\mathrm{CO}_{2}$ geological storage projects. Application of multicriteria decision making, Global NEST Journal, 16(1), 36-40.

Morsi G.W. and El-Hawary M.E. (2010), Novel power quality indices based on wavelet packet transform for nonstationary sinusoidal and non-sinusoidal disturbances, Electric Power Systems Research, 80(7), 753-759.

Patil S., Kasturiwala S., Dahad S. and Jadhav C. (2011), Wavelet tool: Application for human face recognition, International Journal of Engineering Science \& Technology, 3 (3), 2392-2398.

Pedregal D.J., Garcia F.P. and Roberts C. (2010), An algorithmic approach for maintenance management based on advanced state space systems and harmonic regressions, Annals of Operations Research, 166(1), 109-124.

Wang X., Tse P.W., Mechefske C.K. and Hua M. (2010), Experimental investigation of reflection in guided wavebased inspection for characterization of pipeline defects, NDT\&E International, 43, 365-374.

Ye J., He Y., Chen X., Zhai Z., Wang Y. and He Z. (2010), Pipe crack identification based on finite element method of second generation wavelets, Mechanical Systems and Signal Processing, 4(2), 379-393. 